Research Article

\title{
Optimal Control Scheme of the Tethered System for Orbital Transfer under a Constant Thrust
}

\author{
Liang Sun $(\mathbb{D}$, Guowei Zhao $\mathbb{D}$, Hai Huang, and Ming Chen \\ Beihang University, Beijing 100191, China \\ Correspondence should be addressed to Guowei Zhao; zhaoguowei@buaa.edu.cn
}

Received 10 October 2017; Revised 2 February 2018; Accepted 14 February 2018; Published 8 April 2018

Academic Editor: Mahmut Reyhanoglu

Copyright (c) 2018 Liang Sun et al. This is an open access article distributed under the Creative Commons Attribution License, which permits unrestricted use, distribution, and reproduction in any medium, provided the original work is properly cited.

\begin{abstract}
The tethered system with a long tether has its unique advantages in space environment exploration. With the development of requirement, the orbital transfer of the tethered system under a constant thrust and its related optimal control become significant and challenging. Three different optimal control schemes of the tethered system are proposed, including the tension control, the thrust vector control, and the mixed control. In the tension control, in order to ensure the smoothness of pendular motion of the tethered system, different cost functions are adopted and compared. In the thrust vector control, the constraint of thrust direction angle is fully considered. In the mixed control, equivalent conditions to other control schemes are investigated. The advantages and disadvantages of three optimal control schemes are compared and analyzed, which provides a reference for research on the optimal control problem of the tethered system under a constant thrust.
\end{abstract}

\section{Introduction}

In practical application, the tethered system has a broad application prospect in space environment exploration and space resource development [1]. This system is generally formed by a base satellite and a detector joined by a long tether. With the development of requirement, the global spatial distribution of physical parameters needs to be explored, which cannot be obtained only by deploying and retrieving the detector. Thus, the tethered system is facing the problem of orbital transfer under a thrust. In practical engineering, conventional orbital transfers can be utilized, such as the Hohmann orbital transfer and the Bielliptical orbital transfer [2]. During conventional orbital transfers, a certain windlass mechanism needs to be installed on the base satellite for damping out the pendular motion of the tethered system by adjusting the length of a tether, which is essentially consistent with the application of the traditional tethered system on the ellipse orbit. Whereas, this method actually has several limitations in applications. For example, it is not suitable for the tethered system with a short tether due to a sudden impact of impulse thrust; besides, if the windlass mechanism is broken, the pendular motion of the tethered system cannot be inhibited. Thus, a continuous thrust is more appropriate for the orbital transfer of the tethered system, and it can also be implemented to stabilize the attitude of the tethered system. Meanwhile, with the progress of small thrust (microthrust) technology, a small, continuous, and constant thrust becomes a practical and effective method, which possesses merits, such as low control requirements, high safety, and repeatability. Accordingly, proper control must be applied during orbital transfer subject to boundary conditions, cost functions, and path constraints. Therefore, the study on the optimal control of the tethered system under a constant thrust has an important theoretical and practical significance.

In recent five years, the requirement of space debris disposal becomes more urgent and important; thus, research on the tethered system with a thrust is mainly focused on the area of active debris removal (ADR). Aslanov et al. studied the dynamics of large debris connected to a space tug by a tether and also the chaotic motions of tethered satellites with low thrust [3-6]. Jasper and 
Schaub explored an active debris removal method, which utilizes fuel reserves on a recently launched upper stage to rendezvous with and tether to debris [7]. Benvenuto et al. made dynamics analysis and an GNC design of flexible systems for space debris active removal [8]. Linskens and Mooij focused on the preliminary design of a guidance and control system to achieve space-debris removal as well as on the influence of different tether parameters on mission performance [9]. Sun et al. derived analytical solutions of the librational angles; besides, a method of hierarchical sliding mode tension control and a method of thrust control are presented to solve the control problems of the tethered system under a thrust, respectively [10-12]. Huang et al. studied the coupling dynamics modelling of a tethered space robot, explored a space tethered towing method which utilizes thrust to fulfill transfer, and bounded tension to stabilize tether heading; besides, Huang et al. studied the dynamics analysis and a controller design for maneuvering a tethered space net robot and also discussed the design, measurement, control, and experiment of a dexterous tethered space robot [13-16].

In these papers, a continuous thrust is normally implemented during active debris removal; meanwhile, a certain windlass mechanism can be adopted for damping out the pendular motion of the tethered system in orbit maneuvering. Nevertheless, boundary conditions, cost function, and path constraints of the tethered system are not fully considered in the design of a controller. Research on the optimal control of the tethered system under a thrust is limited. Cho and McClamroch proposed an optimal orbit transfer method in which a continuous thrust on the base satellite is controlled to achieve orbital transfer between two circular orbits [17]. In the research, the value of thrust is variable and the length of a tether is fixed; besides, linearized equations are adopted for the control design. Zhong and Zhu proposed a timescale separate optimal control of tethered space-tug systems for space debris removal, in which a Hohmann transfer orbit is implemented [2]. In practical engineering, optimal control methods adopting different actuators present different properties. Based on the previous research, the article is mainly focused on the optimal control of the tethered system under a small, continuous, and constant thrust. In the article, different optimal control schemes are proposed and studied; meanwhile, the advantages and disadvantages of methods are compared and analyzed. Moreover, the effect of cost function and path constraint on control performance is deeply analyzed.

\section{Mathematical Model}

Normally, the base satellite in practical engineering is within the range of $800 \sim 1200 \mathrm{~kg}$. The detectors applied for space environment exploration include a three-dimensional imager, a $\gamma$-ray spectrometer, an X-ray spectrometer, and a microwave radiometer; hence, the mass of the detector is usually within the range of $50 \sim 150 \mathrm{~kg}$. The Kevlar rope is widely used in the tethered system. The length of a tether is within the range of $1 \sim 5 \mathrm{~km}$, and the linear density of a tether $\rho=1 \mathrm{~g} / \mathrm{m}$. With respect to the mass of a detector, the mass of a tether is very small and then is treated as a rigid rod ignoring mass, elastic strain, and damping (a dumbbell model).

It should be noticed that there are several other kinds of tether dynamical models such as the lumped mass model and the beam model, in which the mass, elastic strain, and damping of a tether are fully considered; thus, the deformation of a tether can be described and it also leads to a highorder vibration, which is superimposed on the pendular motion of the tethered system. However, this kind of vibration is limited and does not affect the stability and control of the tethered system. Besides, the dumbbell model is helpful to improve the calculation efficiency of a controller. Thus, a dumbbell model is adopted to derive dynamics equations involved in the control problem (Figure 1).

2.1. Attitude Dynamics. The tethered system is formed by two-end masses joined by a rigid rod of length of $l$. The mass of the base satellite is $M$, and the mass of a detector is $m$. In addition, the base satellite actively drags the detector under a small, continuous, and constant thrust in an orbital plane.

Let $E x^{\prime} y^{\prime} z^{\prime}$ be the geocentric inertial frame. Its origin $E$ is the mass center of the Earth. The axis $E x^{\prime}$ points to the first Aries point, the axis $E y^{\prime}$ is in the equatorial plane, and the coordinate system is right-hand oriented. Subsequently, let Oxyz (Figure 1) be the orbital frame with origin at the mass center of the tethered system $O$. The $O z$ axis points to the mass center of the Earth $E$, the $O x$ axis is normal to the $O z$ axis in the orbital plane and points along the direction of an increasing polar angle $\alpha$, and the coordinate system is right-hand oriented. Afterwards, let $O x_{\mathrm{o}} y_{\mathrm{o}} z_{\mathrm{o}}$ be the original frame with origin at the mass center $O$. The $O z_{\mathrm{o}}$ axis points to the detector, and the noninertial frame is determined by one rotation from the orbital frame $O x y z$. Following the rotations of angle $\theta$ about the $O y$ axis and angle $\phi$ about $O x_{\mathrm{o}}$ axis, $\theta$ and $\phi$ are defined as in-plane and out-of-plane angles, respectively. Compared with the in-plane angle $\theta$, the initial out-of-plane angle $\phi$ is very small; moreover, the coupling of in-plane angle $\theta$ and out-of-plane angle $\phi$ is not obvious during orbital transfer in an orbital plane; thus, for simplicity, the out-of-plane motion is not considered in the study.

Since the method of derivation is similar to that in [8-10], the process of derivation is omitted. According to the Euler-Lagrange equation, the attitude dynamics equations are derived as

$$
\begin{aligned}
& \ddot{l}-l(\dot{\alpha}-\dot{\theta})^{2}+\frac{\mu l}{r^{3}}\left(1-3 \cos ^{2} \theta\right)=\frac{F \sin (\gamma-\theta)}{M}-\frac{T(m+M)}{m M}, \\
& l(\ddot{\alpha}=\ddot{\theta})+2 l(\dot{\alpha}-\dot{\theta})-3 \frac{\mu l}{r^{3}} \sin \theta \cos \theta=\frac{F \cos (\gamma-\theta)}{M},
\end{aligned}
$$

where $\mu=3.986 \times 10^{5} \mathrm{~km}^{3} / \mathrm{s}^{2}$ is the gravitational constant of the Earth. $r$ and $\alpha$ are the geocentric distance and the orbital polar angle of the mass center $O$, respectively. The thrust direction angle $\gamma$ is the intersection angle from $F_{\alpha}$ to $F$. $F$ is the value of thrust $F$, and $F_{\alpha}$ is the transversal component of $F$. Besides, $T$ is the tether tension. 


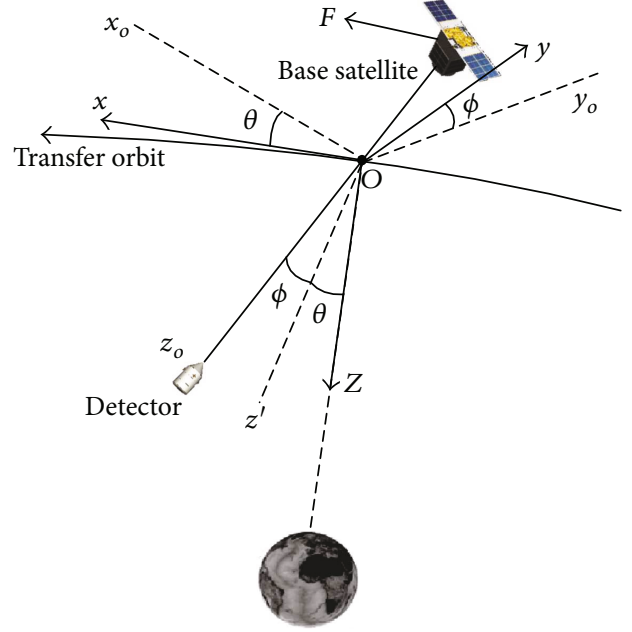

Figure 1: Description of a dumbbell-modeled tethered system.

2.2. Orbit Dynamics. The thrust imposed on the base satellite always remains in the orbital plane. In practical engineering, the value of thrust $F$ is within the range of $100 \mathrm{mN} \sim 10 \mathrm{~N}$ and the specific impulse $I_{\mathrm{s}}$ is in the range of $300 \mathrm{~s} \sim 400 \mathrm{~s}$. Besides, the transfer time usually reaches to $1 \sim 2$ orbit periods; therefore, the fuel consumption approaches to $2 \mathrm{~kg}$ approximately during orbital transfer. With respect to the mass of the base satellite, the fuel consumption can be ignored.

Moreover, because the tethered system is applied for the space environment exploration in the low orbit, the aerodynamic forces can affect the librational motion of the tethered system, especially in a long term. However, the aerodynamic force on the tethered system is smaller than $10^{-5} \mathrm{~N}$. During orbital transfer, the value of thrust $F$ is within the range of $10^{-1} \mathrm{~N} \sim 10 \mathrm{~N}$ and the gravity is greater than $10^{-1} \mathrm{~N}$; hence, the aerodynamic force can be neglected with respect to the thrust and the gravity. The orbit dynamics equations of the tethered system in polar coordinate can be expressed as

$$
\begin{aligned}
\ddot{r}+\frac{\mu}{r^{2}}-\dot{\alpha}^{2} r & =\frac{F \sin \gamma}{M+m}, \\
\ddot{\alpha} r+2 \dot{\alpha} \dot{r} & =\frac{F \cos \gamma}{M+m} .
\end{aligned}
$$

\section{Nonlinear Optimal Control}

3.1. Description of an Optimal Control Problem. During orbital transfer, the base satellite actively drags the detector under a small, continuous, and constant thrust $F$ in the orbital plane. Initially, the attitude of the tethered system is stable without pendular motion, which means $\theta=\dot{\theta}=0$. Then, the thrust $F$ is imposed on the base satellite. The value of thrust is constant, but the direction of thrust can be controlled during orbital transfer; in addition, the tether tension $T$ can also be adjusted. Finally, as the mass center $O$ reaches to the expected

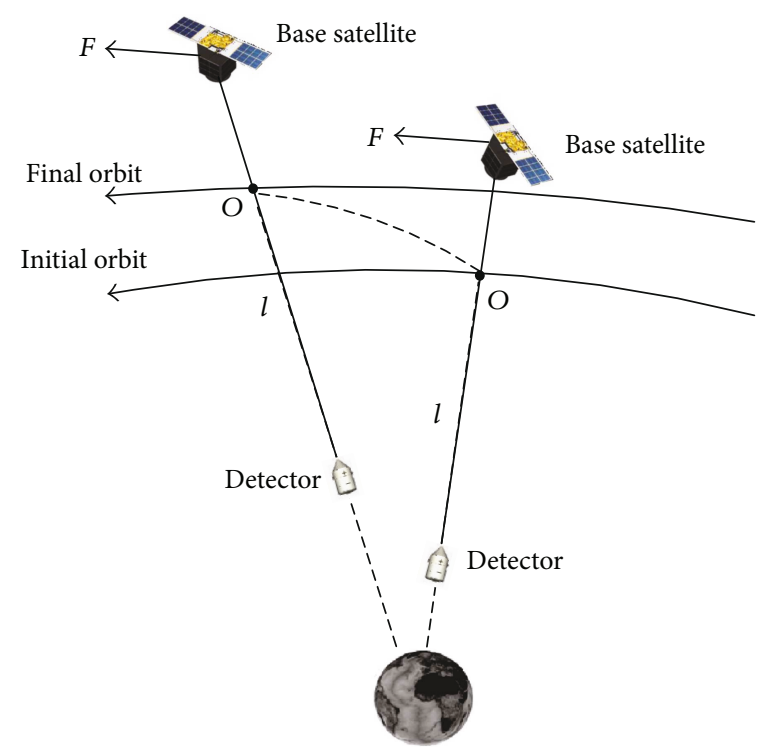

FIgURE 2: Description of an optimal control problem.

circular orbit, the length of a tether comes back to the original value (Figure 2) and $\theta=\dot{\theta}=0$.

3.2. Control Schemes. According to different actuators, optimal control schemes of tethered system under a small, continuous, constant thrust can be divided into three categories.

(1) Tension control: the transfer orbit of the tethered system is ascertained in advance, which is not related to the pendular motion of the tethered system. Besides, the thrust direction angle $\gamma$ is decided by the motion of the mass center; thus, it becomes an independent problem of orbit transfer. During orbital transfer, the tether tension $T$ is controlled to ensure the desired state of the tethered system at the final moment. Moreover, the smoothness of the pendular motion of the tethered system should be taken into consideration, which belongs to an optimal control problem of tether tension.

(2) Thrust vector control: during orbital transfer, the tether of the tethered system is with fixed length. The only control variable is the thrust direction angle $\gamma$, which is requested not only to achieve the orbital transfer between two circular orbits in the shortest possible time but also to guarantee the desired state of the tethered system at the final moment. Besides, to avoid the adverse effect of thrust on the base satellite, the constraint on thrust direction angle $\gamma$ should be considered.

(3) Mixed control: except for the thrust direction angle $\gamma$, the tether tension can also be controlled. These two control variables are applied to achieve the orbital transfer and the desired state of the tethered system in the meantime. 
3.3. Control Method. According to (1) and (2), the dynamic equations of the tethered system under a constant thrust in the orbital plane can be obtained as

$$
\begin{aligned}
& \dot{x}_{1}=x_{2}, \\
& \dot{x}_{2}=f_{1}(x, u), \\
& \dot{x}_{3}=x_{4}, \\
& \dot{x}_{4}=f_{2}(x, u), \\
& \dot{x}_{5}=x_{6}, \\
& \dot{x}_{6}=f_{3}(x, u), \\
& \dot{x}_{7}=x_{8}, \\
& \dot{x}_{8}=f_{4}(x, u),
\end{aligned}
$$

where $x_{1}=l, x_{2}=\dot{l}, x_{3}=\theta, x_{4}=\dot{\theta}, x_{5}=r, x_{6}=\dot{r}, x_{7}=\alpha, x_{8}=\dot{\alpha}$, and the state vector $x$ is defined as $x=\left[\begin{array}{lllll}x_{1} & x_{2} & x_{3} & x_{4} & x_{5}\end{array}\right.$ $\left.\begin{array}{lll}x_{6} & x_{7} & x_{8}\end{array}\right]^{T}$; besides, $u_{1}=\gamma, u_{2}=T$, and the control vector $u$ is defined as $u=\left[u_{1} u_{2}\right]^{T}$. The nonlinear scalar functions of $x$ and $u$ are given by

$$
\begin{aligned}
f_{1}(x, u)= & x_{1}\left(x_{4}-x_{8}\right)^{2}-\mu x_{1} x_{5}^{-3}\left(1-3 \cos ^{2} x_{3}\right) \\
& +F M^{-1} \sin \left(u_{1}-x_{3}\right)-M^{-1} m^{-1}(M+m) u_{2}, \\
f_{2}(x, u)= & -2 x_{1}^{-1} x_{2}\left(x_{4}-x_{8}\right)-2 x_{5}^{-1} x_{6} x_{8}-3 \mu x_{5}^{-3} \sin x_{3} \cos x_{3} \\
& +F(M+m)^{-1} x_{5}^{-1} \cos u_{1}-F m^{-1} x_{1}^{-1} \cos \left(u_{1}-x_{3}\right), \\
f_{3}(x, u)=- & \mu x_{5}^{2}+x_{5} x_{8}^{2}+F(M+m)^{-1} \cos u_{1}, \\
f_{4}(x, u)= & -2 x_{5}^{-1} x_{6} x_{8}+F(M+m)^{-1} x_{5}^{-1} \cos u_{1} .
\end{aligned}
$$

Equation (3) can be directly used for a controller design, as the mixed control is adopted. But if the tension control is implemented, $\gamma$ will be ascertained in advance and the control variable $u=T$; besides, when the thrust vector control is applied, $\dot{l}=0, \ddot{l}=0$, and $u=\gamma$.

According to the description of the optimal control problem, the boundary conditions can be expressed as

$$
\begin{aligned}
& x\left(t_{0}\right)=\left[\begin{array}{llllllll}
l_{0} & 0 & 0 & 0 & r_{0} & 0 & \alpha_{0} & \sqrt{\mu r_{0}^{-3}}
\end{array}\right]^{T}, \\
& x\left(t_{\mathrm{f}}\right)=\left[\begin{array}{llllllll}
l_{\mathrm{f}} & 0 & 0 & 0 & r_{\mathrm{f}} & 0 & \alpha_{\mathrm{f}} & \sqrt{\mu r_{\mathrm{f}}^{-3}}
\end{array}\right]^{T},
\end{aligned}
$$

where $l_{0}$ and $l_{\mathrm{f}}$ are the initial and final lengths of a tether, respectively; besides, $l_{0}=l_{\mathrm{f}}=l_{\mathrm{c}}$ and $l_{\mathrm{c}}$ is the original length of a tether. $r_{0}$ and $r_{\mathrm{f}}$ are the geocentric distances of initial and final circular orbits, respectively. $\alpha_{0}$ and $\alpha_{\mathrm{f}}$ are the initial and final polar angles, respectively.

The inequality path constraint is written as

$$
C(x, u, t) \leq 0
$$

In addition, the cost function (Lagrange type) can be expressed as

$$
J(x, u)=\int_{0}^{t_{\mathrm{f}}} L(x, u, t) d t .
$$

In the study, a MATLAB software, GPOPS (general pseudospectral optimization software) [18], is adopted for solving the optimization control problem. The method employed by GPOPS is the Radau pseudospectral method (RPM) where the collocation points are the Legendre-Gauss-Radau (LGR) points; besides, the hp-adaptive approach is implemented to solve the NLP (nonlinear programming) problem [18].

\section{Case Study}

In this section, three different optimal control schemes are studied, respectively; besides, the effects of the cost function $J(x, u)$ and the path constraint $C(x, u, t)$ on the control performance are analyzed.

4.1. Tension Control Scheme. In the tension control scheme, the thrust direction angle $\gamma$ and the orbital trajectory of the mass center are ascertained in advance. Taking the orbital transfer between two circular orbits as an example, different orbital transfer methods can be adopted, such as an optimized orbital transfer or a transversal orbital transfer. In order to study the optimization of the tether tension, a transversal orbital transfer is implemented, in which the thrust direction angle $\gamma \equiv 0$ during orbital transfer.

According to the transversal orbital transfer, a small, continuous, and constant thrust is imposed on the base satellite and along the transversal direction of the mass center $O$; then, the polar angle $\alpha$ and the geocentric distance $r$ can be expressed as [8]

$$
\begin{aligned}
& \alpha \approx \alpha_{0}+w t-\frac{3 f_{\alpha}}{2 r_{0}} t^{2}-\frac{4 f_{\alpha}}{w^{2} r_{0}} \cos w t+\frac{4 f_{\alpha}}{w^{2} r_{0}}, \\
& r \approx r_{0}+\frac{2 f_{\alpha}}{w} t-\frac{2 f_{\alpha}}{w^{2}} \sin w t,
\end{aligned}
$$

where $\omega=\sqrt{\mu r_{0}^{-3}}$. As $\alpha-\alpha_{0}=2 n \pi(n=1,2 \ldots)$, the orbital trajectory of the mass center returns to an another circular orbit; meanwhile, the transfer time $t_{\mathrm{f}}$ and the transversal thrust acceleration $f_{\alpha}$ can be derived as

$$
\begin{aligned}
t_{\mathrm{f}} & =\frac{2 n \pi}{w}, \\
f_{\alpha} & =\frac{w^{2}\left(r_{\mathrm{f}}-r_{0}\right)}{4 n \pi} .
\end{aligned}
$$

In practical engineering, the mass of the base satellite is within the range of $800 \sim 1200 \mathrm{~kg}$ and the mass of the detector is within the range of $50 \sim 150 \mathrm{~kg}$. The tethered system is mainly utilized to explore the space environment in a low Earth orbit; normally, the orbit altitude is within the range of $400 \sim 800 \mathrm{~km}$. Because the length of a tether is within a limited range of $1 \sim 5 \mathrm{~km}$, the tethered system can only obtain the spatial environment data in the vicinity by deploying and retrieving the detector. In order to obtain the global spatial 
TABLE 1: System parameters of the tethered system under tension control.

\begin{tabular}{lc}
\hline Parameters & Values \\
\hline Number of orbit laps $n$ & 1 \\
Geocentric distance of the initial circular orbit $r_{0}, \mathrm{~km}$ & 7000 \\
Geocentric distance of the final circular orbit $r_{\mathrm{f}}, \mathrm{km}$ & 7010 \\
Transversal thrust acceleration, $\mathrm{m} / \mathrm{s}^{2}$ & $9.25 \times 10^{-4}$ \\
Transfer time $t_{\mathrm{f}}, \mathrm{s}$ & 5835 \\
Mass of the base satellite $M, \mathrm{~kg}$ & 1000 \\
Mass of the detector $m, \mathrm{~kg}$ & 50 \\
Original length of the tether $l_{\mathrm{c}}, \mathrm{m}$ & 1000 \\
Initial value of the in-plane angle $\theta_{0},{ }^{\circ}$ & 0 \\
Final value of the in-plane angle $\theta_{\mathrm{f}},{ }^{\circ}$ & 0 \\
Initial value of the in-plane angle $\theta_{0},{ }^{\circ} / \mathrm{s}$ & 0 \\
Final value of the in-plane angle $\theta_{f},{ }^{\circ} / \mathrm{s}$ & 0 \\
Initial value of tether tension $T_{0}, \mathrm{~N}$ & $3 \mu l_{0} r_{0}^{-3}$ \\
Final value of tether tension $T_{f}, \mathrm{~N}$ & $3 \mu l_{0} r_{\mathrm{f}}^{-3}$ \\
\hline
\end{tabular}

data, the orbit altitude of the tethered system is requested to be increased by at least $10 \mathrm{~km}$. Without loss of generality, the geocentric distance of the initial circular orbit is set to $7000 \mathrm{~km}$ and the geocentric distance of the final circular orbit is set to $7010 \mathrm{~km}$ in the case study. In addition, the other simulation parameters of the tethered system are listed in Table 1.

In the GPOPS [18], the node number $N_{k} \in[6,12]$ and the tolerance is smaller than $10^{-6}$; besides, the tension tether $T>0$. In order to ensure the smoothness of the pendular motion of the tethered system during orbital transfer, the Lagrange function $L=k_{l} \dot{l}^{2}+k_{\theta} \dot{\theta}^{2}$ is adopted. As $k_{l} \neq 0$ and $k_{\theta}=0$, the optimal controller is designed to minimize the variation of tether length. And if $k_{l}=0$ and $k_{\theta} \neq 0$, the variation of the in-plane angle is requested to be the minimum. Besides, when $k_{l} \neq 0$ and $k_{\theta} \neq 0$, both of $\dot{l}^{2}$ and $\dot{\theta}^{2}$ are integrated into account in the Lagrange function. Considering the magnitudes of $\dot{l}^{2}$ and $\dot{\theta}^{2}$ and without loss of generality, the Lagrange function is selected as $L=\dot{l}^{2}, L=\dot{\theta}^{2}$, and $L=1 \times 10^{-3} \dot{l}^{2}+1 \times 10^{3} \dot{\theta}^{2}$, respectively.

As shown in Figure 3, the tethered system returns to the equilibrium position after completing orbital transfer. The curves of $l$ and $\theta$ present approximate symmetrical distributions. Without constraints on $l$ and $\theta$, when the Lagrange function $L=i^{2}$, the length of the tether $l$ changes within the reasonable range of $400 \sim 1000 \mathrm{~m}$ and meanwhile the inplane angle $\theta$ varies in the range of $-48^{\circ} \sim 0^{\circ}$. Though the variation of $\theta$ is large with respect to $L=\dot{\theta}^{2}$, the curve of $\theta$ is smooth and the flight safety can be ensured during orbital transfer. Inversely, if the Lagrange function $L=\dot{\theta}^{2}$, the inplane angle varies in a small range of $-18^{\circ} \sim 0^{\circ}$, but the length of the tether $l$ reaches to $3000 \mathrm{~m}$ and $l$ attains to $\pm 5 \mathrm{~m} / \mathrm{s}$; then, it is negative for the stability of the tethered system and also brings difficulty to the mechanism. Compared with $L=\dot{\theta}^{2}$, the Lagrange function $L=i^{2}$ is conductive to the smoothness of pendular motion and the flight safety. Besides, in order to further improve the performance of optimal control, two methods can be adopted. In the first method, the constraints on $l$ and $\theta$ can be considered, but it may lead to a sudden change of the tether, which is not easy to realize in practical engineering. In the second method, both of $\dot{l}^{2}$ and $\dot{\theta}^{2}$ are integrated into account in the Lagrange function. For instance, as the Lagrange function $L=1 \times 10^{-3} \dot{l}^{2}+1 \times 10^{3}$ $\dot{\theta}^{2}$, with respect to $L=\dot{l}^{2}$, the variation of in-plane angle $\theta$ is reduced to the range of $-35^{\circ} \sim 0^{\circ}$ and meanwhile the curve of $l$ changes a little. In general, this method is more suitable in engineering applications.

Remark 1. In order to improve the calculation efficiency of the controller, the dumbbell model is adopted in this paper. Whereas, in practical engineering, though the effect of tether vibration on the pendular motion is limited, several effects associated with the tether flexibility, elasticity, and the detector rotation, such as "bounce back" and "tail wagging," should be taken into account. In order to soften this problem, two methods can be adopted. One method is to introduce the lumped model instead of the dumbbell model, which can improve the control accuracy; but even so, the qualitative trends, results, and conclusions are consistent. The other method is to guarantee the good initial condition of the tether by utilizing the windlass mechanism; then, the "bounce back" phenomenon can be avoided.

Remark 2. In the tension control scheme, the tension is defined as the control input, which is provided by a windlass mechanism; whereas, there is no actuator that can directly provide the desired time-varying tension signal. In practical engineering, the tension of the tether can be measured by a force sensor and transmitted to a motor controller. The controller drives the motor to operate, and then the desired tension of the tether can be provided. It should be noticed that the error, delay, and transmission of tether tension ought to be fully considered in the feedback controller.

Remark 3. GPOPS generates the open-loop control, and the control inputs are obtained online. In order to ensure the closed-loop stability and the robustness to disturbances, the real-time merits of the presented schemes can be further exploited to design a closed-loop controller based on more advanced control design techniques such as modelpredictive control.

4.2. Thrust Vector Control. In the thrust vector control scheme, the thrust direction angle $\gamma$ can be adjusted, but the length of the tether is with fixed length. In order to make a comparison, the value of thrust acceleration is still selected as $9.25 \times 10^{-4} \mathrm{~m} / \mathrm{s}^{2}$ and other simulation parameters are consistent with Table 1 . The Lagrange function $L$ is set as 1 and $\dot{\theta}^{2}$, respectively. When $L=1$, the transfer time is requested to 


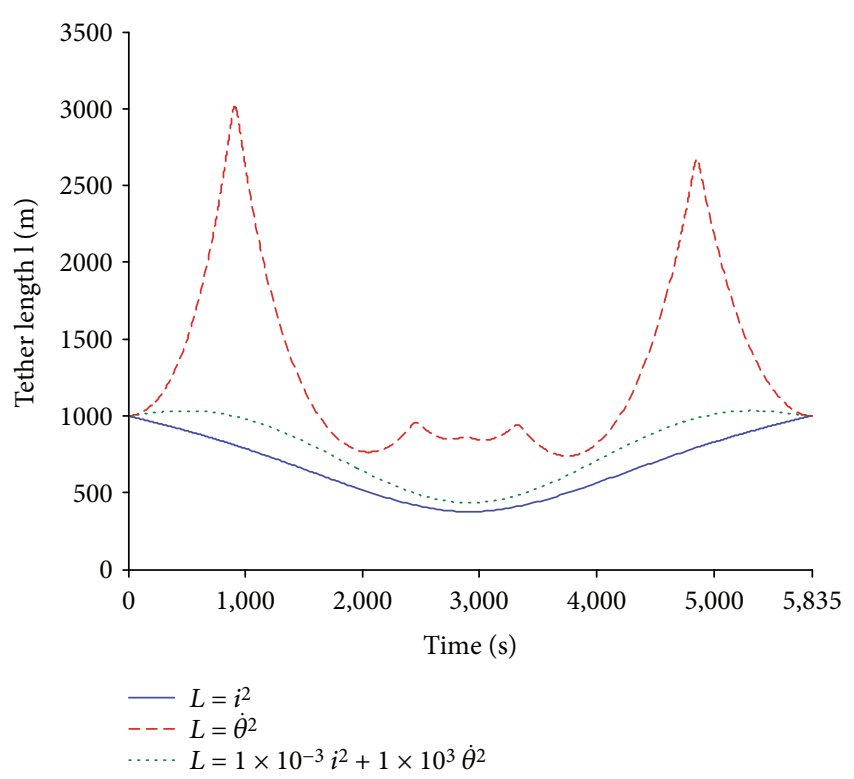

(a) Tether length $l$

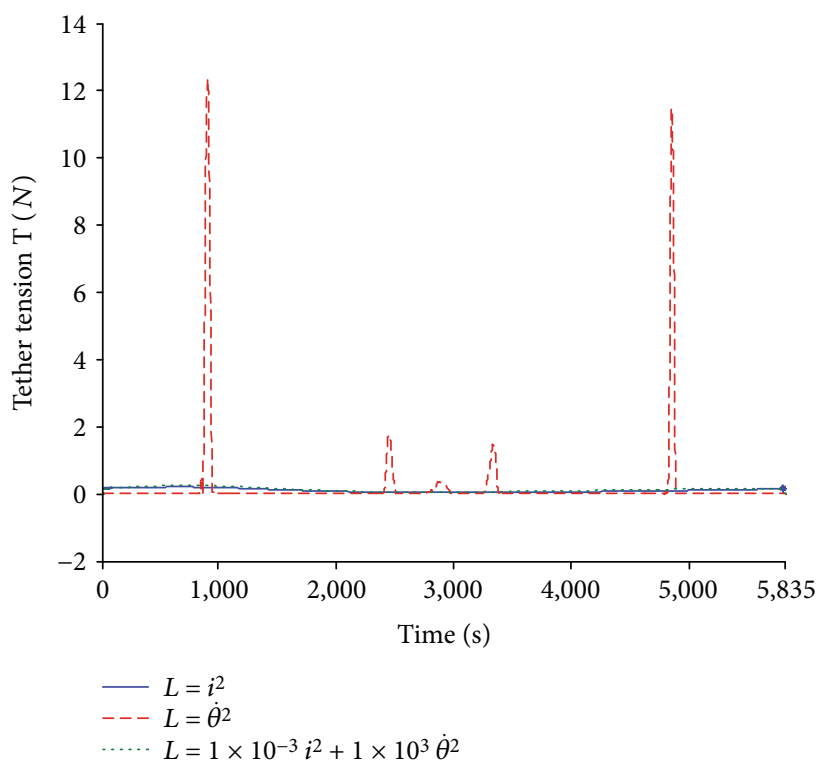

(c) Tether tension $T$

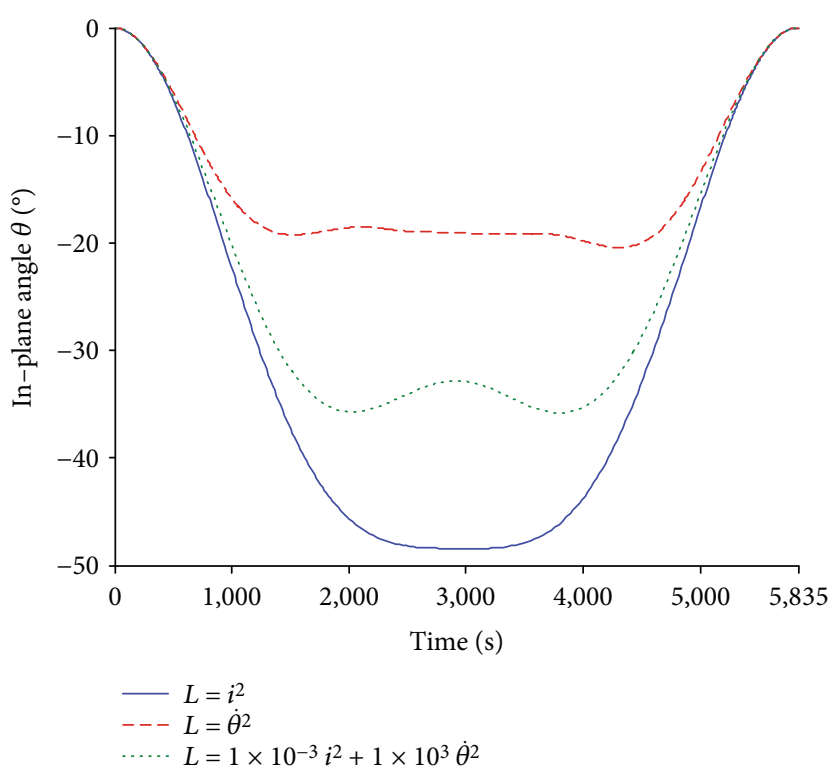

(b) In-plane angle $\theta$

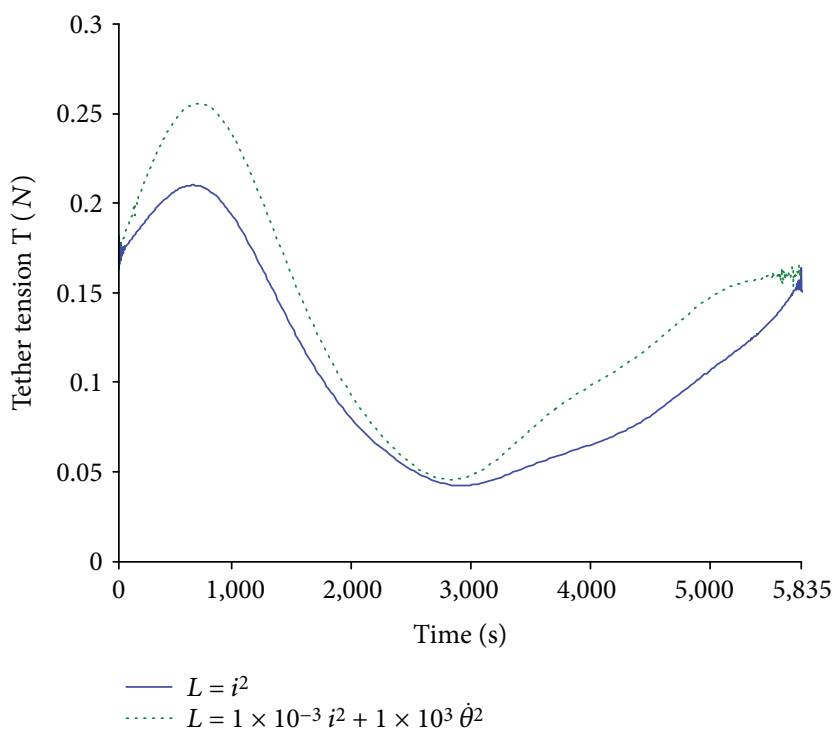

(d) Tether tension $T$ (enlarged view)

FIGURE 3: Time responses of the tethered system under tension control.

be shortest; when $L=\dot{\theta}^{2}$, the variation of the in-plane angle is requested to be the minimum.

As shown in Figure 4, when the Lagrange function $L=1$, the thrust direction angle $\gamma$ changes in the range of $-15^{\circ} \sim 55^{\circ}$. Obviously, as $\gamma$ is utilized to control the orbital transfer and the pendular motion at the same time, the transfer time $t_{\mathrm{f}}$ between two circular orbits is $6214 \mathrm{~s}$, which is longer than the transfer time of the transversal thrust transfer orbit (5835 s). Meanwhile, the in-plane angle $\theta$ varies within the range of $-30^{\circ} \sim 0^{\circ}$ and the tether tension maintains in the range of $0.02 \sim 0.12 \mathrm{~N}$. Though the variation of $\theta$ is large with respect to $L=\dot{\theta}^{2}$, the curve of $\gamma$ is smooth and the controller can be realized in practical engineering. To be opposite, if the
Lagrange function $L=\dot{\theta}^{2}$, the in-plane angle varies in a small range of $-7^{\circ} \sim 0^{\circ}$, but the transfer time reaches to $16,419 \mathrm{~s}$ and $\gamma$ changes dramatically, which goes against the flight safety during orbital transfer.

Thus, $L=\dot{\theta}^{2}$ is not suitable for the optimal control of the tethered system during orbital transfer.

Furthermore, in order to avoid the adverse effect of thrust on the base satellite, the constraint on thrust direction angle $\gamma$ is considered when $L=1$; then, the constraints of $|\gamma| \leq 40^{\circ}$, $|\gamma| \leq 50^{\circ}$, and $|\gamma| \leq 60^{\circ}$ are selected, respectively. As shown in Figure 5, if $|\gamma| \leq 60^{\circ}$, the inequality path constraint does not affect the optimized result. Whereas, if more strict constraint 


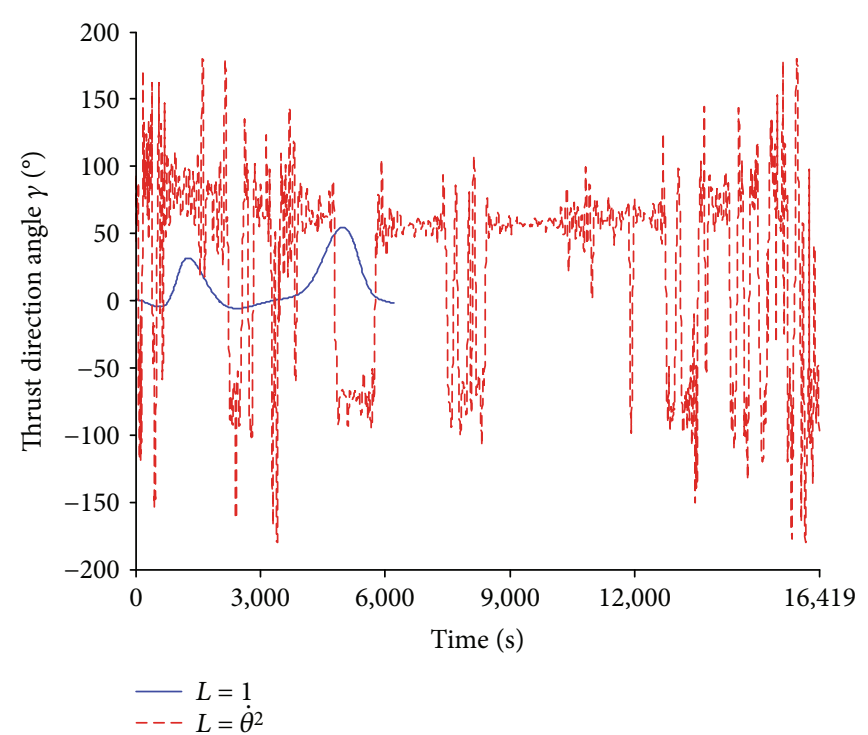

(a) Thrust direction angle $\gamma$

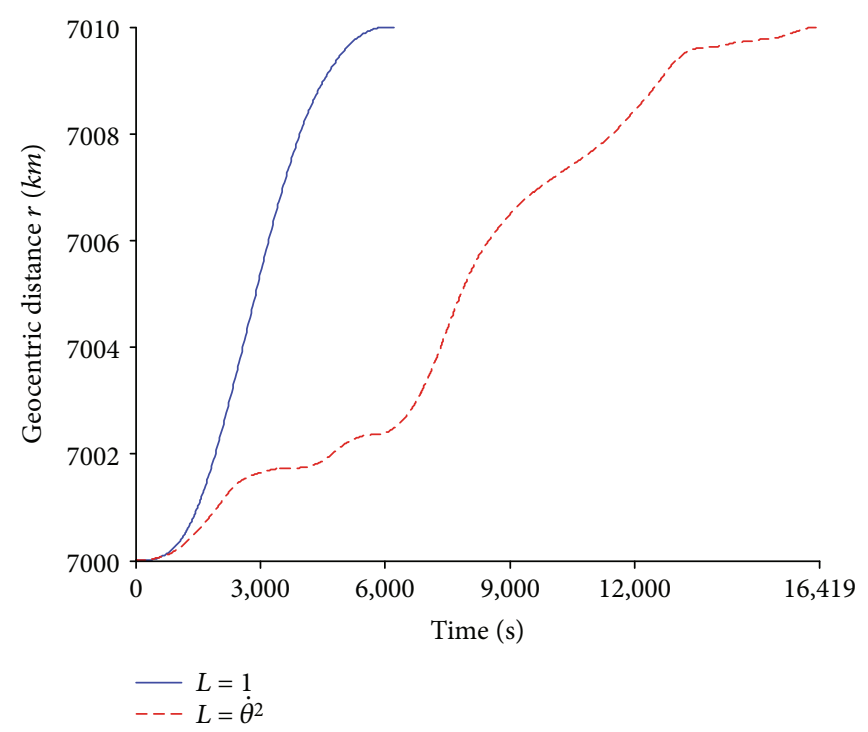

(c) Geocentric distance $r$

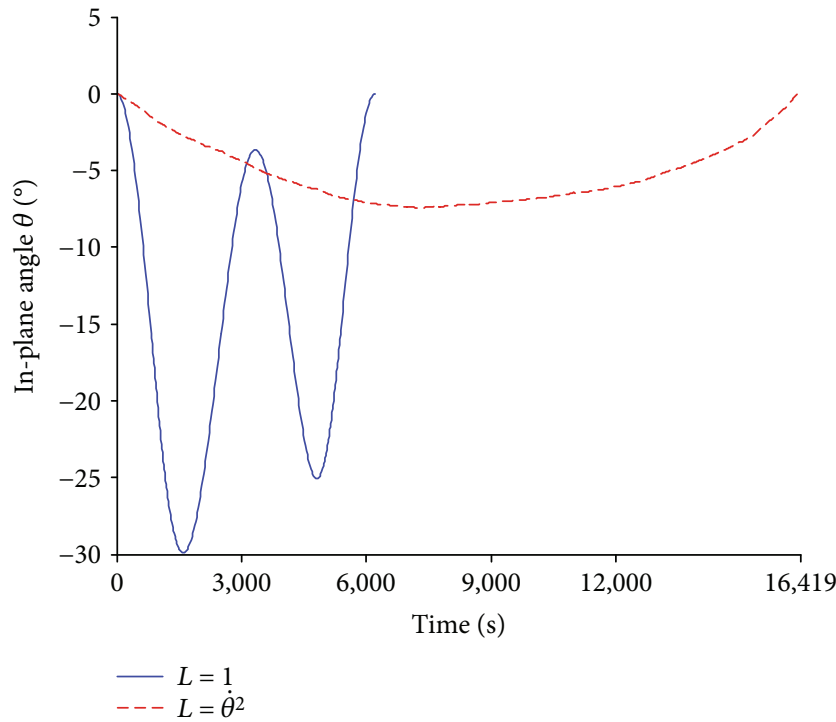

(b) In-plane angle $\theta$

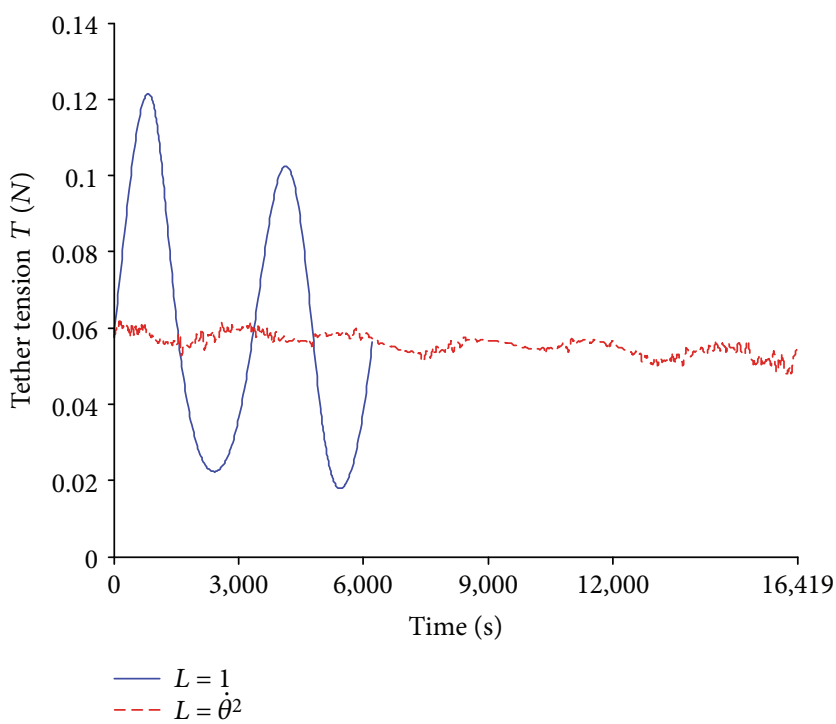

(d) Tether tension $T$

FIGURE 4: Time responses of the tethered system under thrust control.

of $|\gamma| \leq 40^{\circ}$ is considered, the transfer time rises to $6228 \mathrm{~s}$, but $\theta$ and $T$ change a little; thus, proper constraint on $\gamma$ is effective and conducive to orbital transfer. However, if the threshold value of $|\gamma|$ continues to decrease or even tends to zero, $\gamma$ will switch back and forth and reasonable optimal solutions cannot be obtained. Moreover, in order to study the effect of the thrust on the optimal control, the thrust accelerations $f=9 \times 10^{-4} \mathrm{~m} / \mathrm{s}^{2}, f=9.25 \times 10^{-4} \mathrm{~m} / \mathrm{s}^{2}$, and $f=10 \times 10^{-4} \mathrm{~m} / \mathrm{s}^{2}$ are selected, respectively (Figure 6). Accordingly, the final moments of orbital transfer are $5909 \mathrm{~s}, 6214 \mathrm{~s}$, and $6358 \mathrm{~s}$, respectively. Obviously, when the thrust acceleration $f$ increases, the transfer time $t_{\mathrm{f}}$ is shortened; whereas, the variations of the in-plane angle $\theta$ and the tether tension $T$ increase. In practical engineering, the value of thrust is decided by the specific task, but the trends of time responses present were similar.

4.3. Mixed Control. In the mixed control scheme, both $\gamma$ and $T$ are implemented to solve the problem of optimal control. Similarly, the value of thrust acceleration is selected as $9.25 \times 10^{-4} \mathrm{~m} / \mathrm{s}^{2}$ and other simulation parameters are consistent with Table 1; besides, the tension tether $T>0$.

At first, the Lagrange function $L$ is set to 1 . As shown in Figure 7, when the transfer time is requested to be the shortest, the thrust is implemented only for orbit optimization; thus, the transfer time $t_{\mathrm{f}}$ decreases to $5821 \mathrm{~s}$ and the trajectory of the orbit is not related to the pendular motion of the tethered system. In other words, when 


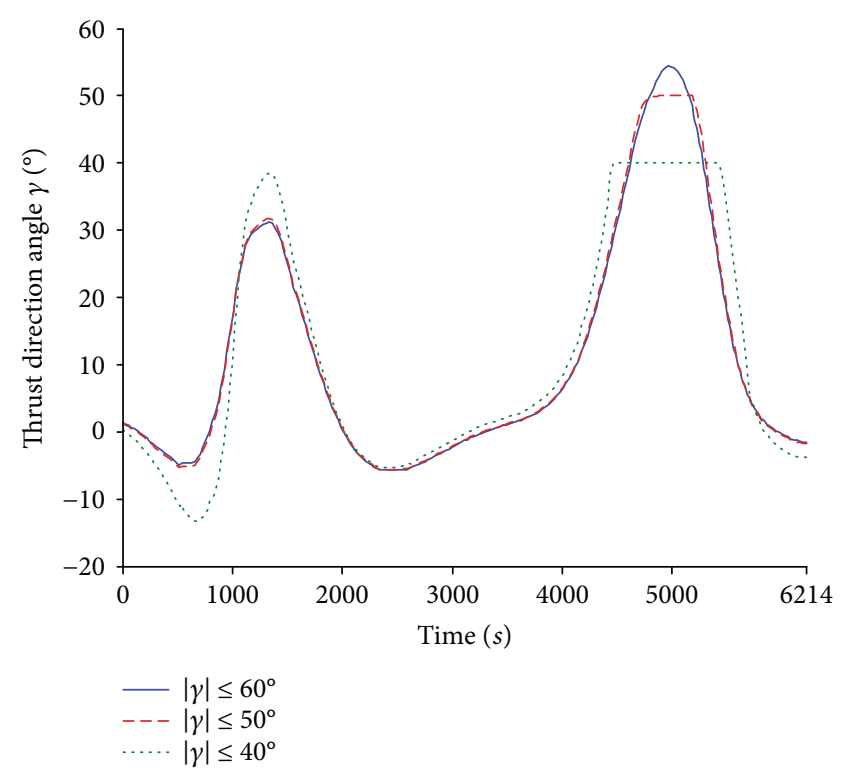

(a) Thrust direction angle $\gamma$

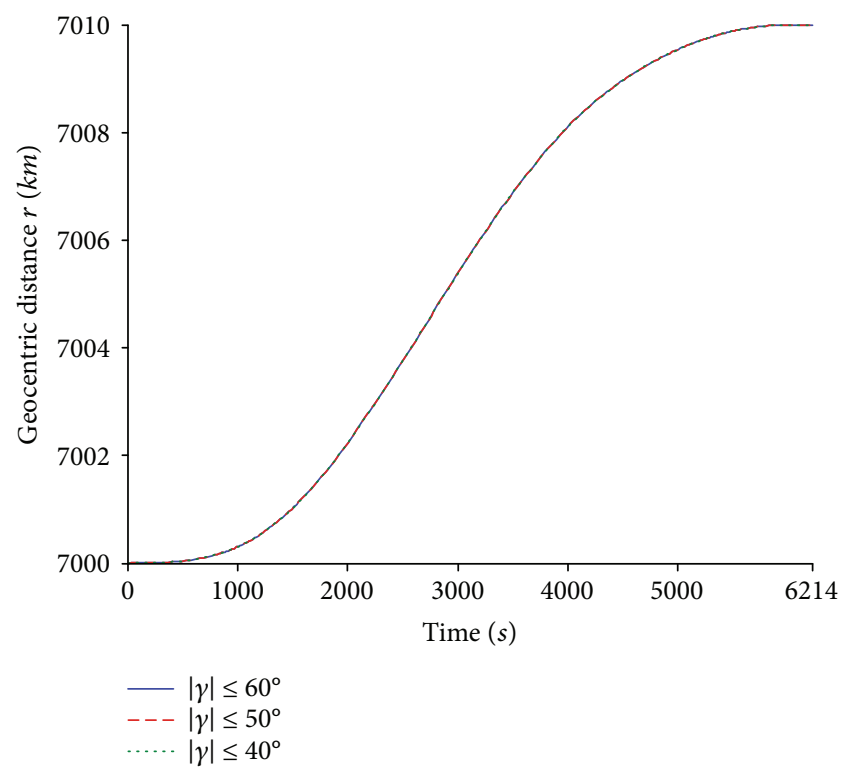

(c) Geocentric distance $r$

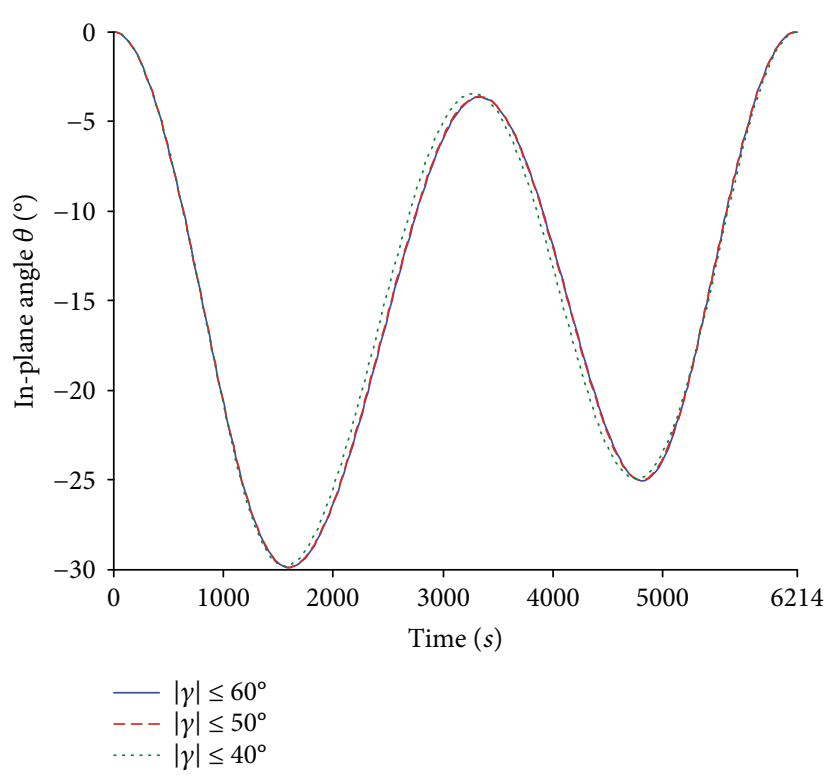

(b) In-plane angle $\theta$

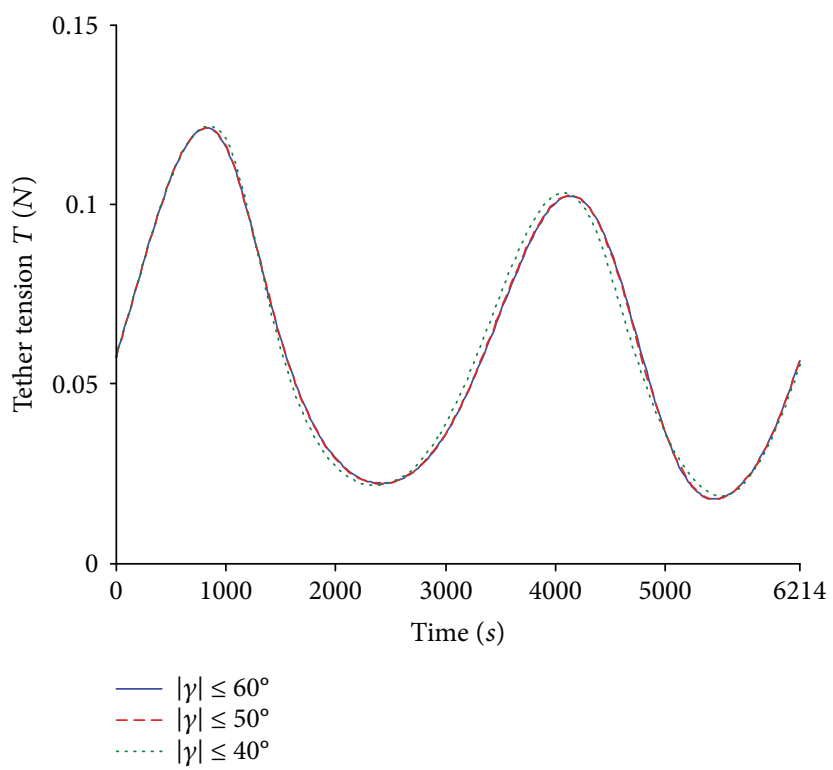

(d) Tether tension $T$

FIGURE 5: Time responses of the tethered system with different constraints on $\gamma$.

$L=1$, the mixed control is same as the tension control in essence except for the difference that a transversal thrust transfer orbit is replaced by an optimized transfer orbit. During this optimized orbital transfer, the tethered system can be viewed as a spacecraft with no tethers.

Moreover, the Lagrange function $L$ is set to $\dot{l}^{2}, \dot{\theta}^{2}$, and $1 \times 10^{-2} \dot{l}^{2}+1 \times 10^{2} \dot{\theta}^{2}$, respectively. As shown in Figure 8 , when $L=\dot{l}^{2}$, the tether length is fixed at $1000 \mathrm{~m}$ and the transfer time attains to $6932 \mathrm{~s}$; meanwhile, the in-plane angle $\theta$ varies within the range of $-21^{\circ} \sim 0^{\circ}$ and the tether tension changes mildly. Actually, when $L=i^{2}$, the mixed control is almost same as the thrust vector control but the transfer time is not requested to be the shortest. If the Lagrange function $L=\dot{\theta}^{2}$, the in-plane angle $\theta$ is limited to $-2^{\circ} \sim 0^{\circ}$; however, the length of the tether reaches to $10,000 \mathrm{~m}$, the transfer time approaches to $14,737 \mathrm{~s}$, and the thrust direction angle $\gamma$ changes seriously. Obviously, $L=\dot{l}^{2}$ is adverse to the orbital transfer. If $L=1 \times 10^{-2} \dot{l}^{2}+1 \times 10^{2} \dot{\theta}^{2}$, the in-plane angle $\theta$ decreased to the range of $-17^{\circ} \sim 0^{\circ}$ with respect to $L=\dot{l}^{2}$; in the meantime, the transfer time $t_{\mathrm{f}}$ changes to $7360 \mathrm{~s}$ and the variation of tether length is only $1.5 \mathrm{~m}$. Overall, the mixed control has both advantages of tension control and thrust vector control; besides, the performance can be improved by adjusting the Lagrange function. Therefore, the mixed control is more practical and effective in engineering. 


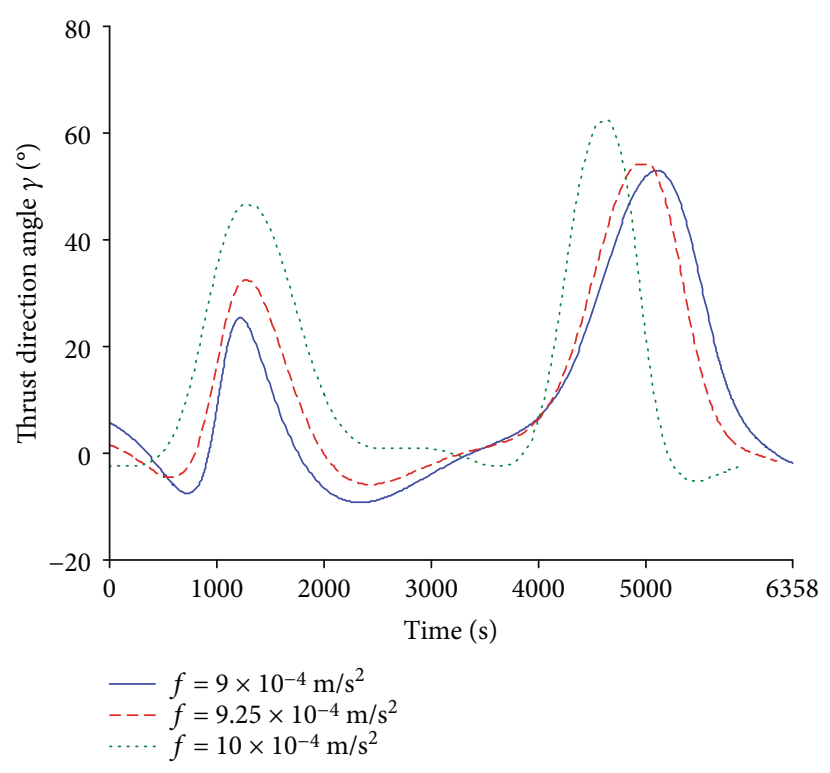

(a) Thrust direction angle $\gamma$

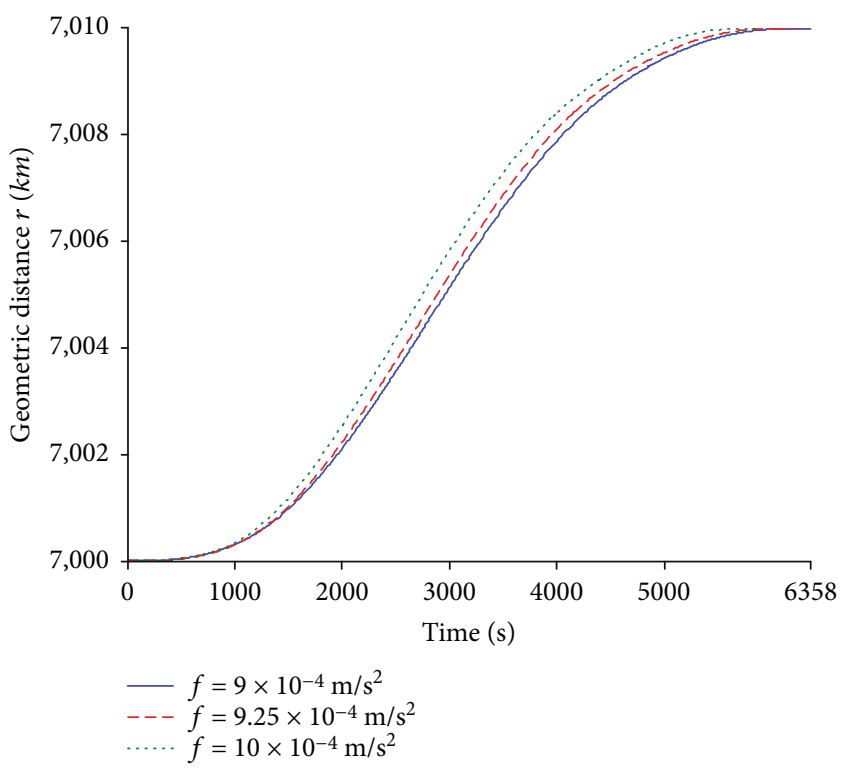

(c) Geocentric distance $r$

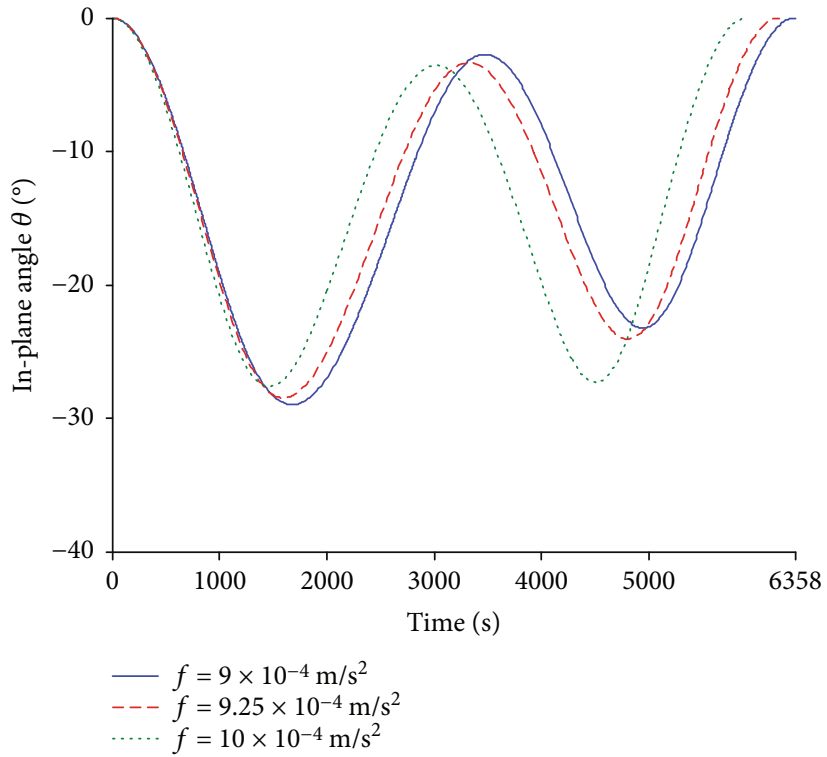

(b) In-plane angle $\theta$

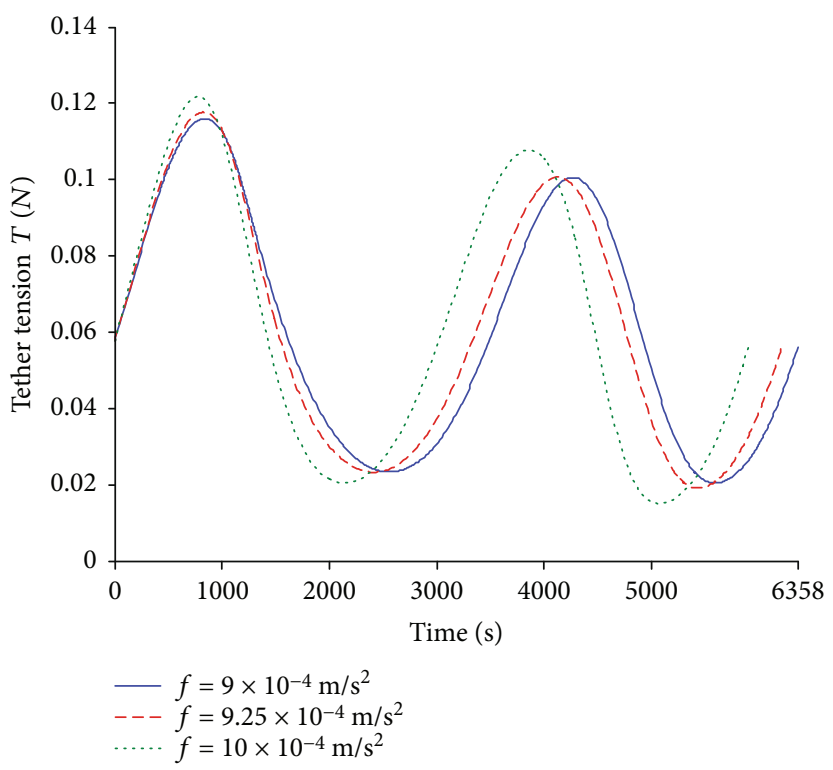

(d) Tether tension $T$

FIGURE 6: Time responses of the tethered system with different thrust accelerations.

\section{Conclusion}

Based on a series of analyses and simulations of optimal control of the tethered system, the following conclusions can be drawn: in practical engineering applications, all three optimal control schemes possess their own merits. In terms of the tension control, the orbit trajectory of the mass center of the tethered system is ascertained in advance without regard to the pendular motion of the tethered system, so typical transfer orbits can be implemented, such as the timeoptimal transfer orbit, the transversal thrust transfer orbit, and the Hohmann transfer orbit. Furthermore, the smoothness of pendular motion can be ensured by adopting a proper cost function. In the tension control, the windlass mechanism can help avoid the slackness of the tether. Accordingly, the reliability of the windlass mechanism needs to be further verified in space applications. In the thrust vector control scheme, the only control variable is the thrust direction angle $\gamma$, which is adopted for optimizations of both orbit and attitude of the tethered system. This method is convenient due to its simple configuration of the actuator, but the fuel consumption is higher with respect to the tension control; besides, though the constraint of $\gamma$ is considered, the magnitude of variation of thrust direction angle $\gamma$ is still large, which will increase the control difficulty of the base satellite. As far as the mixed control is concerned, two control 


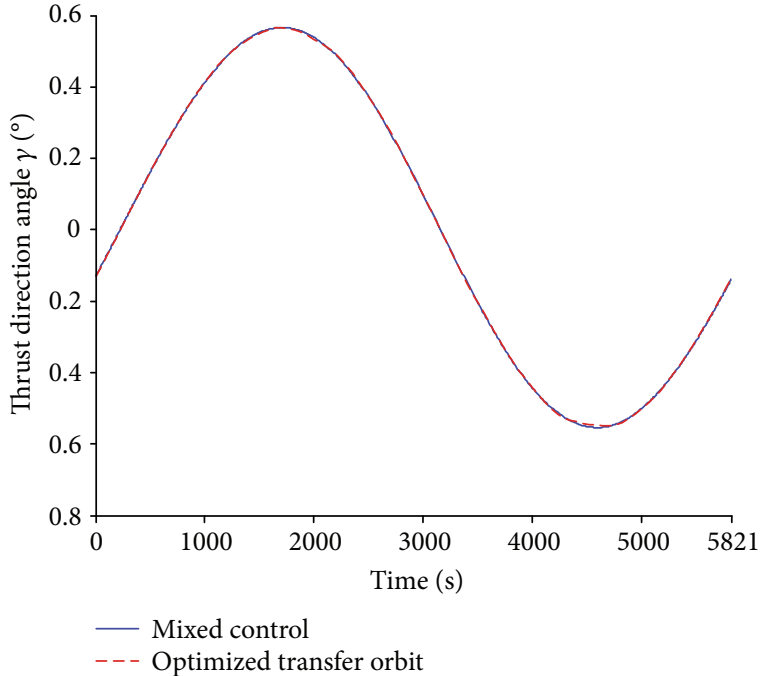

(a) Thrust direction angle $\gamma$

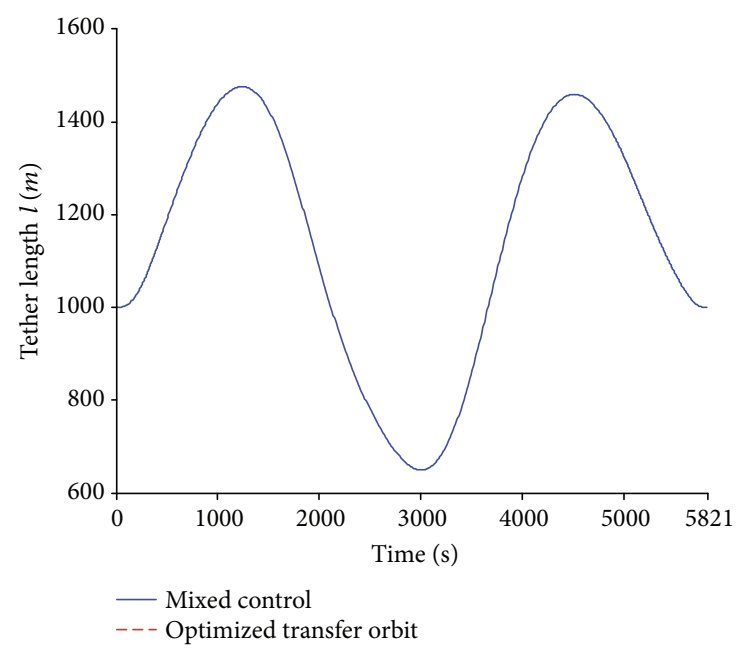

(c) Tether length $l$

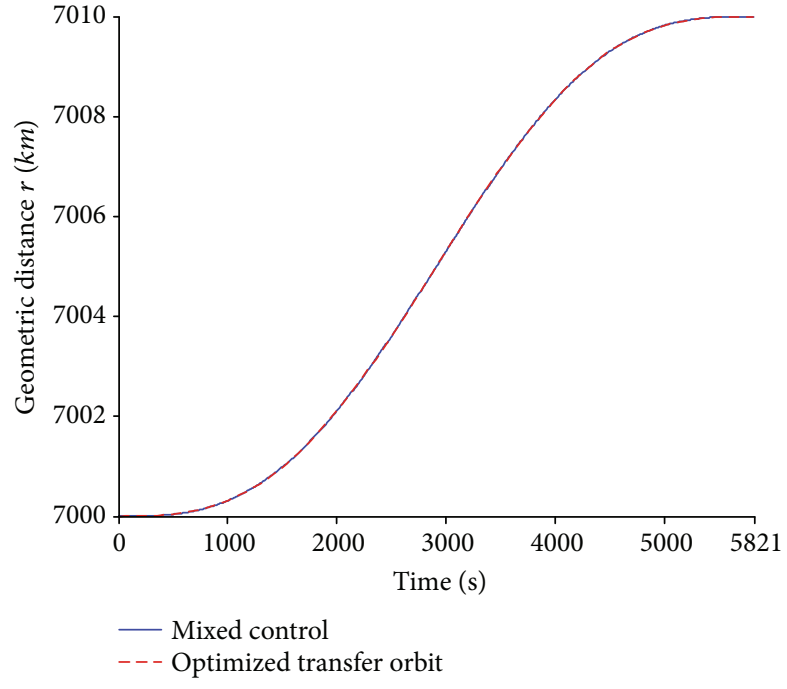

(b) Geocentric distance $r$

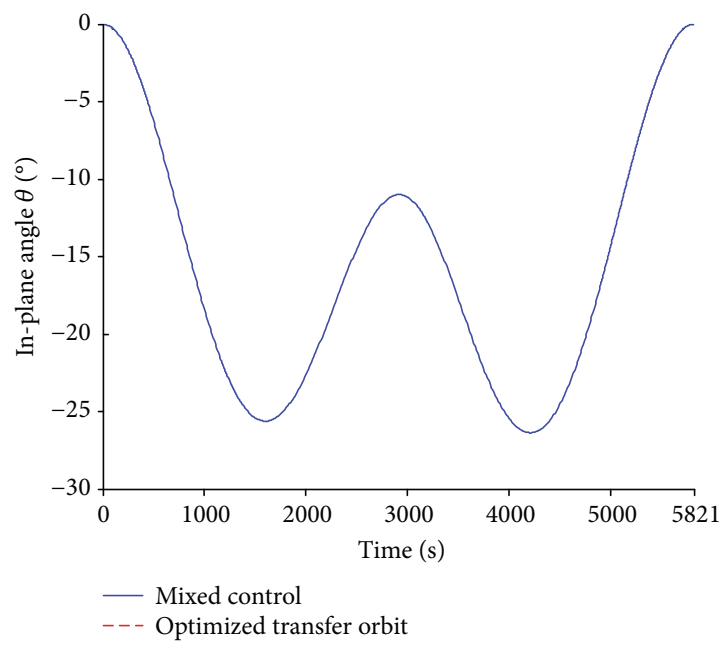

(d) In-plane angle $\theta$

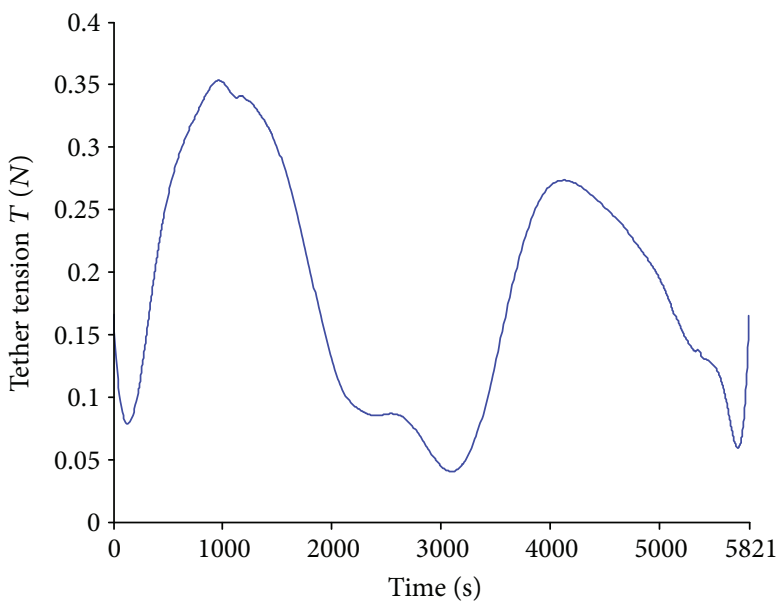

- Mixed control

- - - Optimized transfer orbit

(e) Tether tension $T$

Figure 7: Time optimal control of the tethered system under mixed control. 


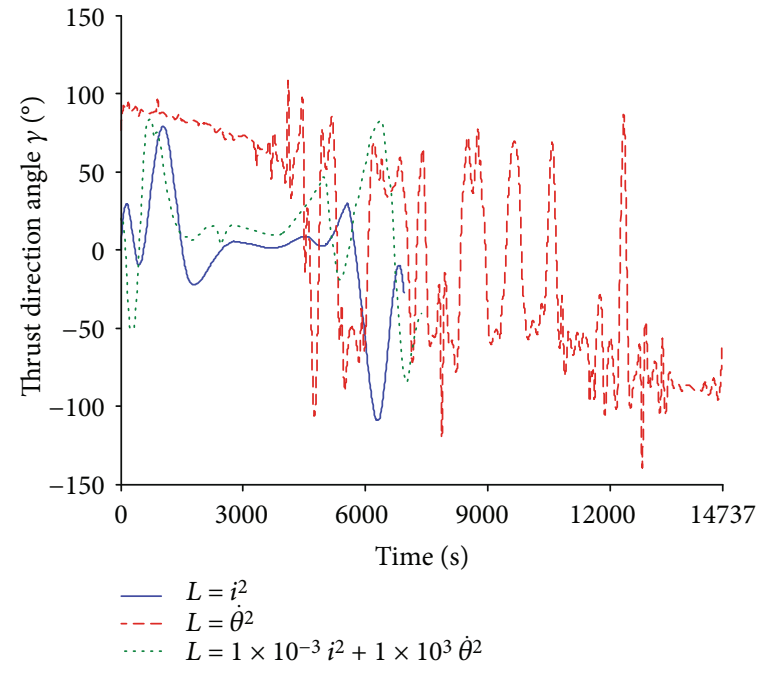

(a) Thrust direction angle $\gamma$

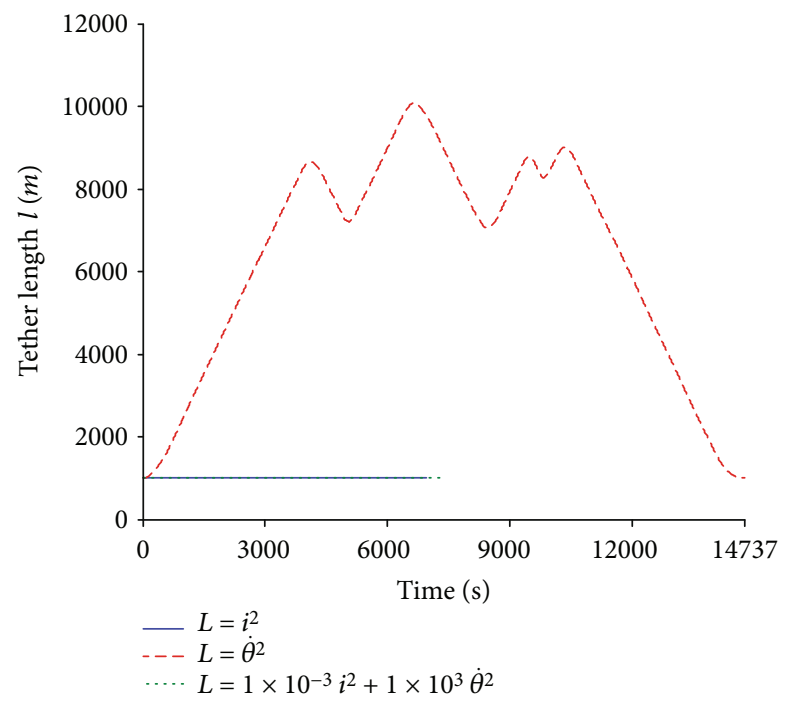

(c) Tether length $l$

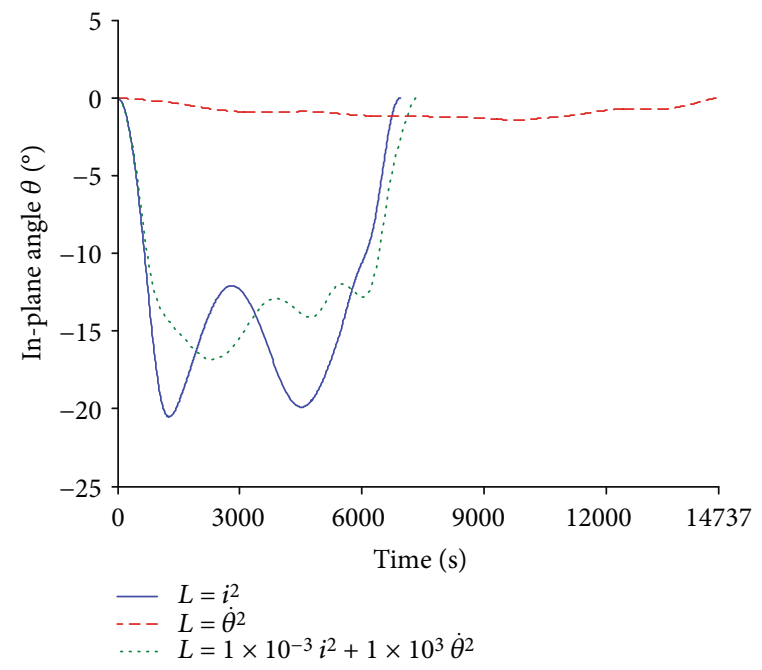

(e) In-plane angle $\theta$

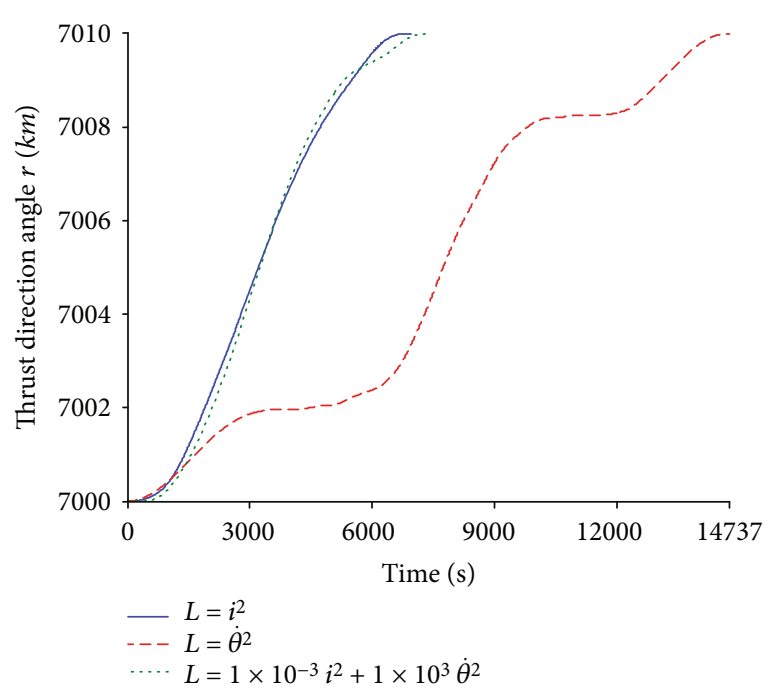

(b) Geocentric distance $r$

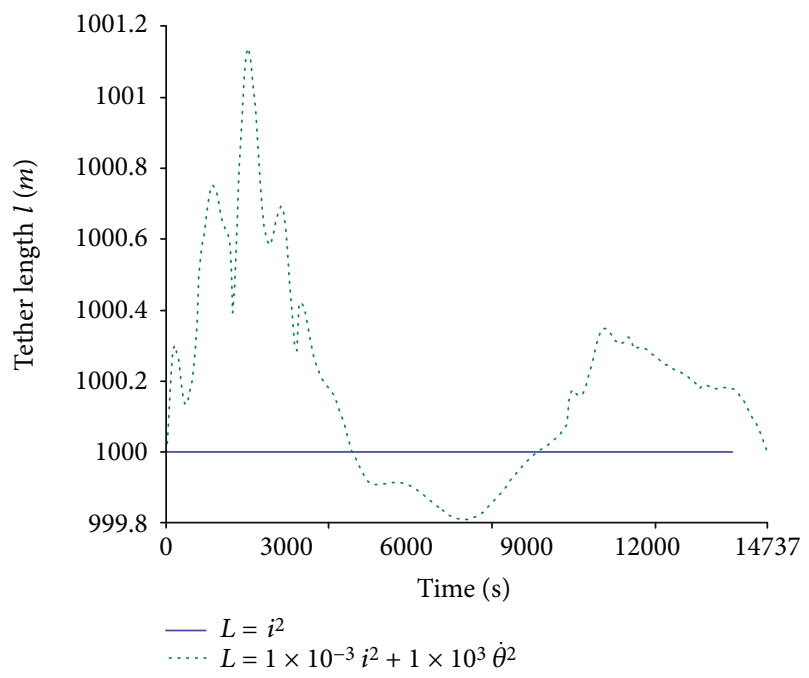

(d) Tether length $l$ (enlarged view)

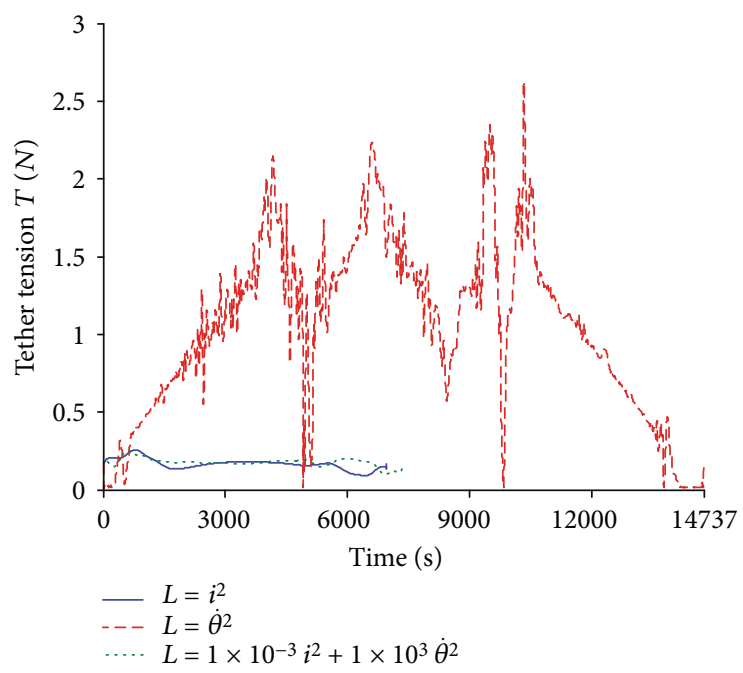

(f) Tether tension $T$

FIgURE 8: Time responses of the tethered system under mixed control. 
variables $T$ and $\gamma$ are employed; hence, this method combines the advantages of both tension control and thrust vector control on the performance and the practicability. The mixed control is best to be utilized as the main control mode; whereas, as the fuel consumption is limited or the windlass mechanism is broken, the control mode can be switched to the tension control mode or thrust vector control mode. According to the optimal performance, the Lagrange function $L=\dot{\theta}^{2}$ is not suitable for optimal control; inversely, $L=k_{l} \dot{l}^{2}+k_{\theta} \dot{\theta}^{2}$ is conducive to the orbital transfer, but the values of $k_{l}$ and $k_{\theta}$ need to be further studied in practical engineering and as well as the threshold value of $\gamma$. This paper provides a reference for the research on the optimal control problem of the tethered system under a constant thrust.

\section{Conflicts of Interest}

There is no conflict of interest in the manuscript.

\section{Acknowledgments}

This research is funded by the National Natural Science Foundation of China under Grant no. 11602008 and Grant no. 11572016, which do not lead to any conflict of interests regarding the publication of this manuscript.

\section{References}

[1] Y. Chen, R. Huang, L. He, X. Ren, and B. Zheng, "Dynamical modelling and control of space tethers: a review of space tether research," Nonlinear Dynamics, vol. 77, no. 4, pp. 1077-1099, 2014.

[2] R. Zhong and Z. H. Zhu, "Timescale separate optimal control of tethered space-tug systems for space-debris removal," Journal of Guidance, Control, and Dynamics, vol. 39, no. 11, pp. 2540-2545, 2016.

[3] V. S. Aslanov and V. V. Yudintsev, "Dynamics of large debris connected to space tug by a tether," Journal of Guidance, Control, and Dynamics, vol. 36, no. 6, pp. 1654-1660, 2013.

[4] V. Aslanov and V. Yudintsev, "Dynamics of large space debris removal using tethered space tug," Acta Astronautica, vol. 91, no. 10, pp. 149-156, 2013.

[5] V. Aslanov, A. Misra, and V. Yudintsev, "Chaotic motions of tethered satellites with low thrust," in 67th International Astronautical Congress (IAC), Guadalajara, Mexico, September 2016.

[6] V. Aslanov and V. Yudintsev, "Dynamics of large space debris removal using tethered space tug," Acta Astronautica, vol. 91, pp. 149-156, 2013.

[7] L. Jasper and H. Schaub, "Input shaped large thrust maneuver with a tethered debris object," Acta Astronautica, vol. 96, no. 4, pp. 128-137, 2014.

[8] R. Benvenuto, S. Salvi, and M. Lavagna, "Dynamics analysis and GNC design of flexible systems for space debris active removal," Acta Astronautica, vol. 110, pp. 247-265, 2015.

[9] H. T. K. Linskens and E. Mooij, "Tether dynamics analysis and guidance and control design for active space-debris removal," Acta Astronautica, vol. 107, pp. 150-162, 2015.

[10] G. Zhao, L. Sun, S. Tan, and H. Huang, "Librational characteristics of a dumbbell modeled tethered satellite under small, continuous, constant thrust," Proceedings of the Institution of Mechanical Engineers, Part G: Journal of Aerospace Engineering, vol. 227, no. 5, pp. 857-872, 2013.

[11] L. Sun, G. Zhao, and H. Huang, "Stability and control of tethered satellite with chemical propulsion in orbital plane," Nonlinear Dynamics, vol. 74, no. 4, pp. 1113-1131, 2013.

[12] G. Zhao, L. Sun, and H. Huang, "Thrust control of tethered satellite with a short constant tether in orbital maneuvering," Proceedings of the Institution of Mechanical Engineers, Part G: Journal of Aerospace Engineering, vol. 228, no. 14, pp. 2569-2586, 2014.

[13] P. Huang, Z. Hu, and Z. Meng, "Coupling dynamics modelling and optimal coordinated control of tethered space robot," Aerospace Science and Technology, vol. 41, pp. 36-46, 2015.

[14] B. S. Wang, Z. J. Meng, and P. F. Huang, "A towing orbit transfer method of tethered space robots," in 2015 IEEE International Conference on Robotics and Biomimetics (ROBIO), pp. 964-969, Zhuhai, China, December 2015.

[15] Z. Meng, B. Wang, and P. Huang, "A space tethered towing method using tension and platform thrusts," Advances in Space Research, vol. 59, no. 2, pp. 656-669, 2017.

[16] F. Zhang and P. Huang, "Releasing dynamics and stability control of maneuverable tethered space net," IEEE/ASME Transactions on Mechatronics, vol. 22, no. 2, pp. 983-993, 2017.

[17] S. Cho and N. H. McClamroch, "Optimal orbit transfer of a spacecraft with fixed length tether," Journal of Astronautical Sciences, vol. 51, no. 2, pp. 195-204, 2003.

[18] C. L. Darby, W. W. Hager, and A. V. Rao, "An $h p$-adaptive pseudospectral method for solving optimal control problems," vol. 32, no. 4, pp. 476-502, 2011. 


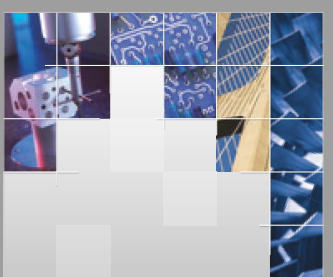

\section{Enfincering}
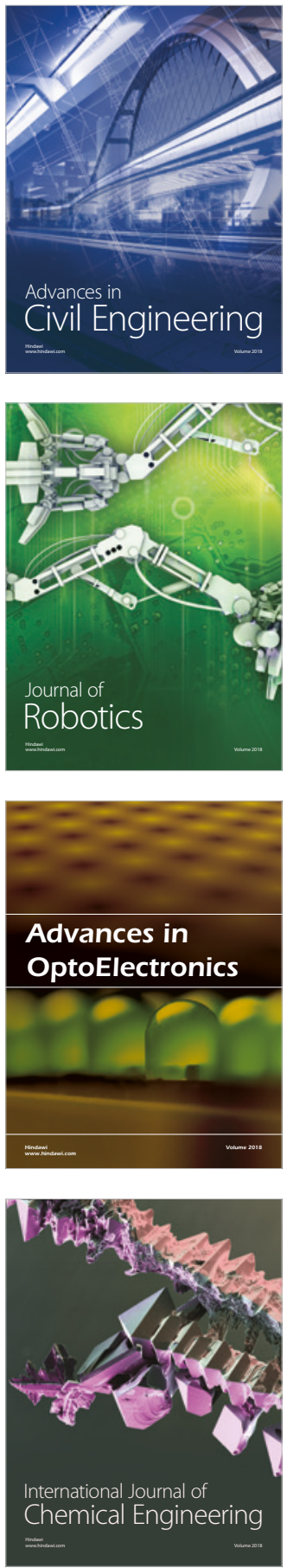

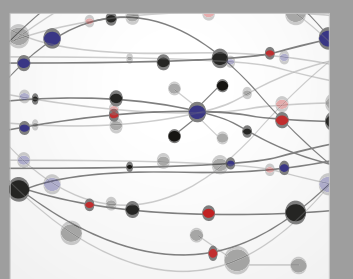

\section{Rotating \\ Machinery}

The Scientific World Journal

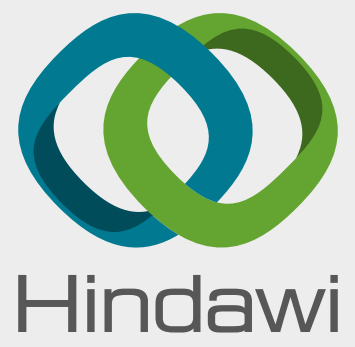

Submit your manuscripts at

www.hindawi.com
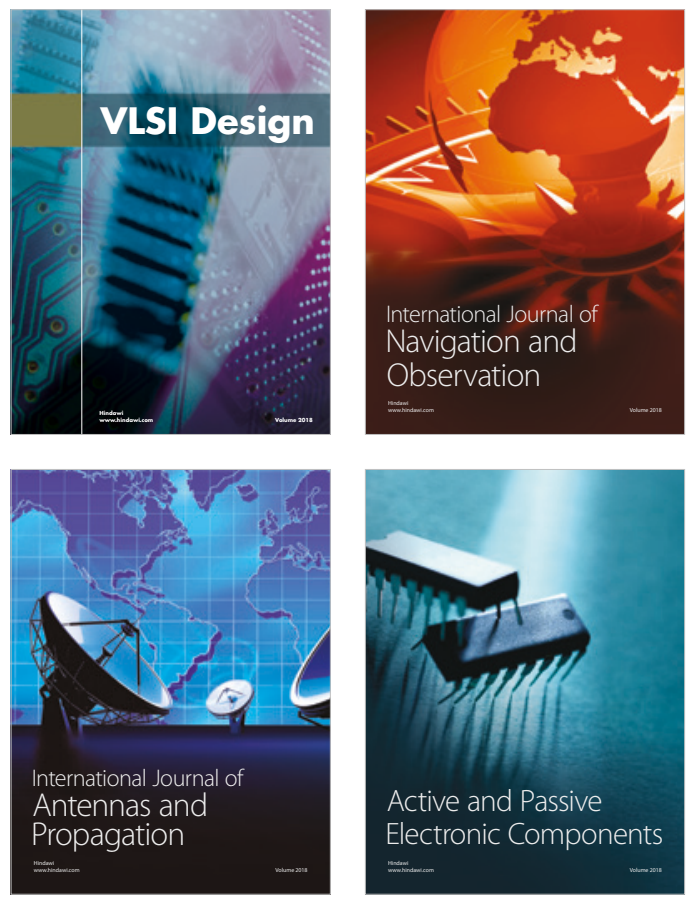
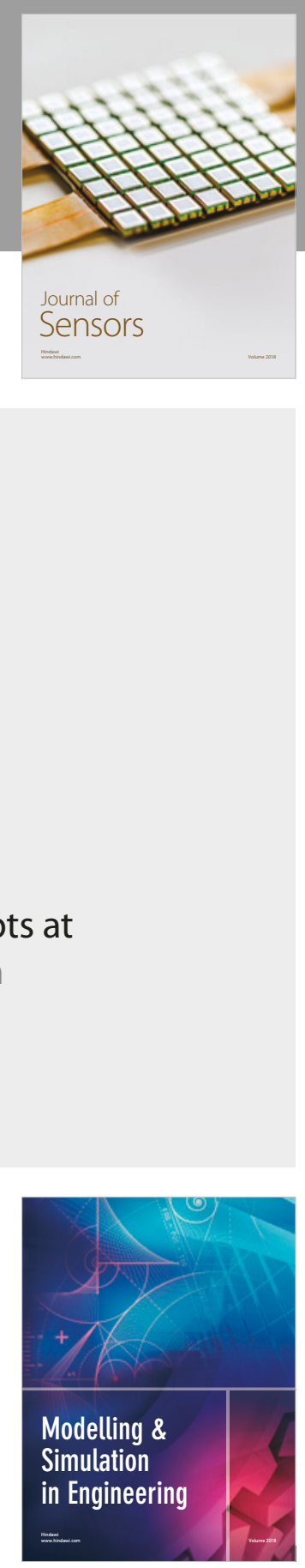

\section{Advances \\ Multimedia}
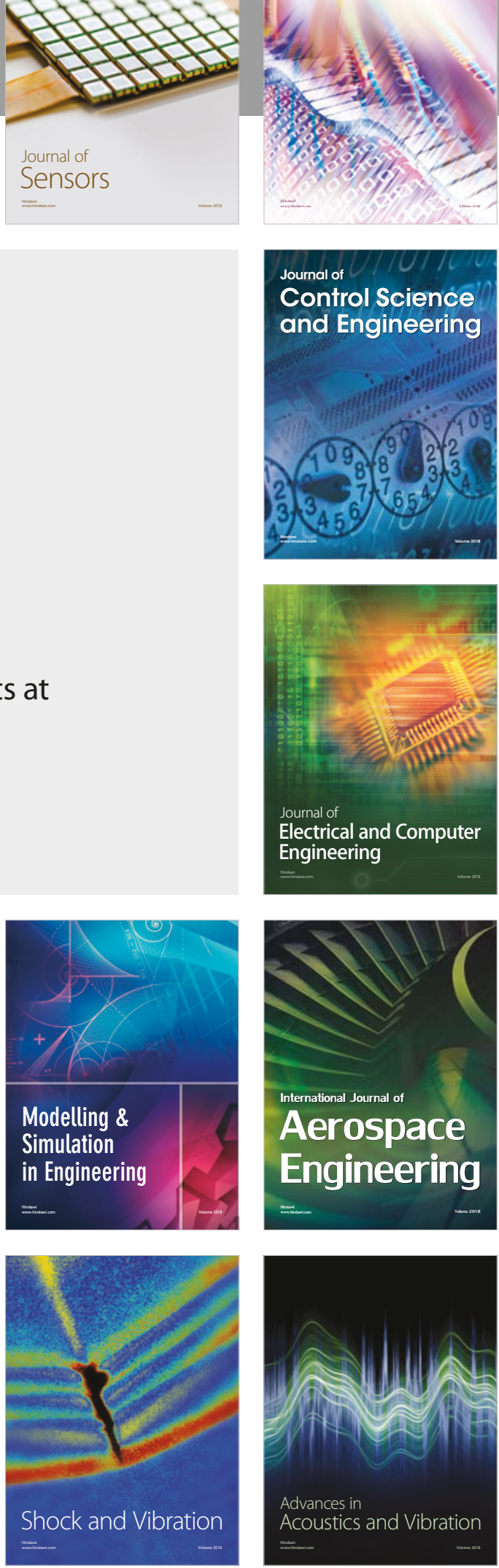